\title{
Topology Optimisation for High Frequency Vibration Energy Harvesting
}

\author{
David J. Munk ${ }^{a}$, Ethan J. G. Ellul ${ }^{b}$ and Scott D. Moss ${ }^{c^{*}}$ \\ Defence Science and Technology Group, Aerospace Division, 506 Lorimer Street, Fishermans \\ Bend, Victoria, Australia, 3207 \\ adavid.munk@dst.defence.gov.au, bethan.ellul@dst.defence.gov.au, \\ cscott.moss@dst.defence.gov.au \\ *Corresponding author. Tel.: +61-3-9626-7958; fax: +61-3-9626-7089.
}

(C) 2020 Commonwealth of Australia

\section{Keywords: Vibration Energy Harvesting, Topology Optimisation, Helicopter Gearbox}

\begin{abstract}
Topology optimisation has been used to design a piezoelectric energy harvester capable of harvesting the vibration present on a helicopter gearbox. The gearbox vibrations, with frequencies in the kilo-hertz range and having amplitudes of $10-100 g$ (where $g=9.81 \mathrm{~m} / \mathrm{s}^{2}$ ), are generated by gear-meshing within the transmission. These accelerations, large in amplitude and high in frequency, are ideal sources for vibration energy harvesting, with the harvested power potentially used to power autonomous condition-based-maintenance systems. This paper will discuss the first and simplest of the harvesters that were designed and manufactured, i.e. a $0.51 \mathrm{~mm}$ thick spring steel cantilever that uses a Pz27 piezoceramic transducer, which is sensitive to $1900 \mathrm{~Hz}$ gearbox vibrations and can produce $300 \mu \mathrm{W}$ from a $2 g$ host acceleration.
\end{abstract}

\section{Introduction}

Downtime for routine maintenance and checks, in both commercial and military environments, can leave equipment out of operation for extended periods. While this maximises operational safety, it also comes with a potentially costly reduction in availability. Structural Health Monitoring systems (SHMs) can minimise this downtime by providing an ongoing assessment of structural condition, permitting the detection and monitoring of safety-critical degradation [1,2].

Powering SHMs can be problematic, with these systems often being placed in difficult to access positions and unable to be powered locally. A potential solution is the pairing of lowpower SHMs with energy harvesters, siphoning ambient vibrational energy in the environment and converting it into usable electrical energy. One well-studied method of capturing waste mechanical energy is via piezoelectric-based vibrational energy harvesting (PEH) [3]. PEH devices based on traditional piezoceramics $\mathrm{Pb}\left[\mathrm{Zr}_{\mathrm{x}} \mathrm{Ti}_{1-\mathrm{x}}\right] \mathrm{O}_{3}$ (PZT) [4] have been thoroughly explored over the past two decades, with recent reviews indicating that power densities of 10$100 \mathrm{~mW} / \mathrm{cm}^{3}$ are achievable [5,6]. This paper will discuss a high-frequency PEH prototype device designed using a recently developed topology optimisation algorithm, known as Bidirectional Evolutionary Structural Optimisation (BESO), to tune the device to the principal gear-meshing frequency of a Bell 206B-1 Kiowa helicopter main rotor transmission at $1900 \mathrm{~Hz}$ [6].

Topology optimisation has been identified as the most challenging and economically rewarding task in structural design [7]. Generally speaking, topology optimisation seeks to find the best location to place structure in a given design domain subjected to predefined objectives, constraints, loads and boundary conditions. The main advantage of topology optimisation over 
other structural optimisation techniques is that no specified initial structural topology needs to be presumed a priori. Over the last few decades, since the introduction of material distribution methods [8], topology optimisation has been rapidly developed into a design tool used by the engineering industry [9][10][11].

The optimal design of structures subjected to vibration was first considered in the 1970s in the form of shape optimisation with respect to the fundamental and higher-order eigenfrequencies of transversely vibrating beams [12]. Many studies have considered dynamic stability as the objectives [13] where it is advantageous to keep the natural frequencies of the structure away from any driving frequencies. Ultimately, the goal of this work is to develop an in-situ energy harvesting device that can utilise these frequencies to power a low-power condition based SHM system to monitor a helicopter during flight. This paper discusses the modelling, optimisation, and testing of such a device under laboratory conditions, being exposed to accelerations of up to 2.0g. These results are then compared to initial models, and the feasibility for use on the helicopter transmission is discussed.

\section{Principles of Operation}

As mentioned in the introduction, the principal gear-meshing frequency of a Bell 206B-1 Kiowa helicopter main rotor transmission is $1900 \mathrm{~Hz}$ with other significant vibration at $2250 \mathrm{~Hz}$ and $2500 \mathrm{~Hz}$ [6]. The aim of the device reported in this paper is to harvest vibrational energy at the dominant $1900 \mathrm{~Hz}$ frequency - the cantilevered topology was optimised to be sensitive to this frequency. This is the first step in designing a PEH that is sensitive to all three of the main meshing-frequencies, work that will be reported elsewhere. The harvester discussed in this paper, shown schematically in Fig. 1, is a topology optimised cantilever with a piezoceramic disk transducer, the combination of which is tuned to $1900 \mathrm{~Hz}$. The topology optimised design was waterjet-cut from spring steel and secured between two steel plates to form the root. Finite element modelling was used to find the region of highest in-plane stress, upon which the PZT disk was mounted using silver epoxy.

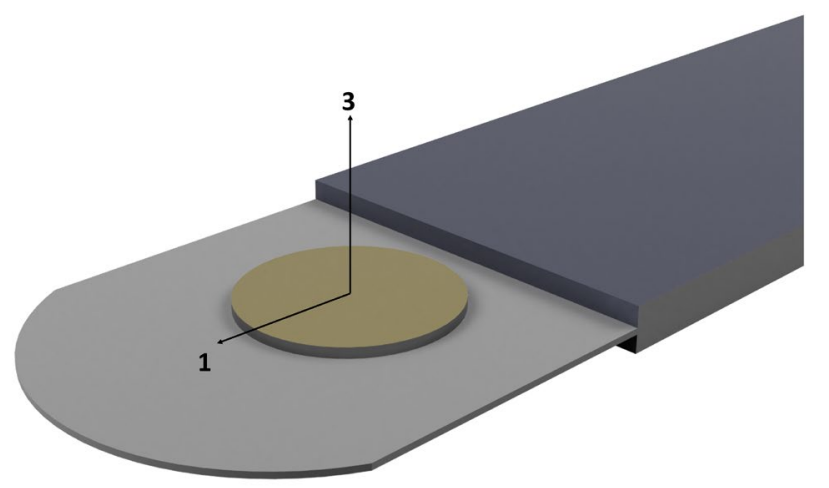

Figure 1. Schematic of the piezoelectric energy harvester showing the thin topology optimised cantilever manufactured from spring steel, with the thicker root section, and the PZT disk transducer with crystalline orientation.

\section{Theory}

The aim of this study is to design a cantilever energy harvesting device that leverages the available vibration energy in a helicopter gearbox. Accelerometer data has indicated that the 
largest $g$-load on the gearbox being considered, that of a Bell 206B-1 Kiowa helicopter, is $12 g$ and occurs at a frequency of $1900 \mathrm{~Hz}$ [6]. Investigations to date have shown that the resonance frequency of the harvesting device plays a key role in the device's power output [14]. Furthermore, the mass of the energy harvesting device is proportional to the stored mechanical energy. This study implements a topology optimisation algorithm to tune the resonance frequency of a cantilever energy harvesting device to the vibration frequency of the maximum $g$ load from a Bell 206B-1 Kiowa helicopter gearbox while keeping the volume of the structure as high as possible. Therefore, the design problem can be mathematically defined as follows:

maximise: $V$

subject to: $\omega_{1}=1900$

where $V$ and $\omega_{1}$ are the volume and fundamental frequency of the energy harvesting device, respectively. Therefore, the optimisation algorithm finds the structural topology that has the largest volume with a fundamental frequency of $1900 \mathrm{~Hz}$. In this study, a recently developed Bidirectional Evolutionary Structural Optimisation (BESO) algorithm is used to solve the design problem [15]. The BESO algorithm developed in [15] replaces the traditional heuristic optimality criteria method with an integer-linear program (ILP) and, thus, enables the handling of multiple constraints. Nevertheless, the method is evolutionary in the sense that only gradual design changes are permitted in each iteration. Therefore, a neighbourhood constraint must be employed to ensure that only small design changes in each iteration are made. The BESO algorithm uses the finite element formulation to solve the physics of the problem. Hence, the design problem can be formulated as an optimisation problem as follows:

$$
\begin{aligned}
& \text { maximise: } V(\boldsymbol{x}) \\
& \text { subject to: }\left([K]-\omega_{n}^{2}[M]\right) \phi_{n}=0 \\
& \omega_{1}=1900 \\
& \begin{array}{l}
\sum_{i \in \boldsymbol{x}=0}^{\omega_{1}} \Delta x_{i} \leq \eta_{a} \cdot N \\
\sum_{i \in \boldsymbol{x}=1} \Delta x_{i} \geq-\eta_{r} \cdot N
\end{array} \\
& x_{i} \in\{0,1\} \quad \forall i \in[1, N]
\end{aligned}
$$

where $\boldsymbol{x}$ is the design variable vector and $x_{i}$ is the $i^{\text {th }}$ design variable, $[K]$ and $[M]$ are the global stiffness and mass matrices of the structure, $\omega_{n}$ and $\phi_{n}$ are the natural mode frequency and shape of the structure, $\eta_{a}$ and $\eta_{r}$ determine the maximum number of elements added and removed in each iteration and $N$ is the number of design variables. The binary design variable $x_{i}$ physically represents the density of the $i^{\text {th }}$ element; where, in this study, a hard-kill BESO method [16] is employed. The BESO method solves the discrete problem, resulting in clear structural boundaries. Therefore, the resulting topologies can be manufactured by traditional methods or additively if complex topologies result.

In order to guarantee that a solution to the optimisation problem exists, some restrictions on the resulting design must be introduced [17]. Experience has shown that filtering of the sensitivity function is a highly efficient way to ensure mesh in-dependency [18]. Furthermore, it has been shown to give results that are stable under mesh refinement and maintains a minimum 
length scale. In this study, a filter scheme is used to smooth the element sensitivity numbers across the entire domain [17].

As mentioned, the optimisation algorithm translates the physical equations into the finite element formulation to solve the physics of the problem. Therefore, to determine the natural frequencies and mode shapes of the structure, the undamped and unloaded equations of motion must be solved. The equations of motion are defined as follows:

$$
[M] \ddot{x}+[K] x=0
$$

where $\boldsymbol{x}$ is the displacement vector of the structure. To solve the above equation a harmonic solution, i.e. $x=\phi_{n} \cdot \sin \left(\omega_{n} \cdot t\right)$, is assumed. By implementing the harmonic solution in the equations of motion the following eigenvalue problem results:

$$
\left([K]-\omega_{n}^{2}[M]\right) \phi_{n}=0
$$

which can be solved for both the natural frequencies, $\omega_{n}$, and modes shapes, $\phi_{n}$, respectively. The finite element model and the first mode shape for the optimised cantilever, having a fundamental frequency of $1900 \mathrm{~Hz}$, is illustrated in Fig. 2.
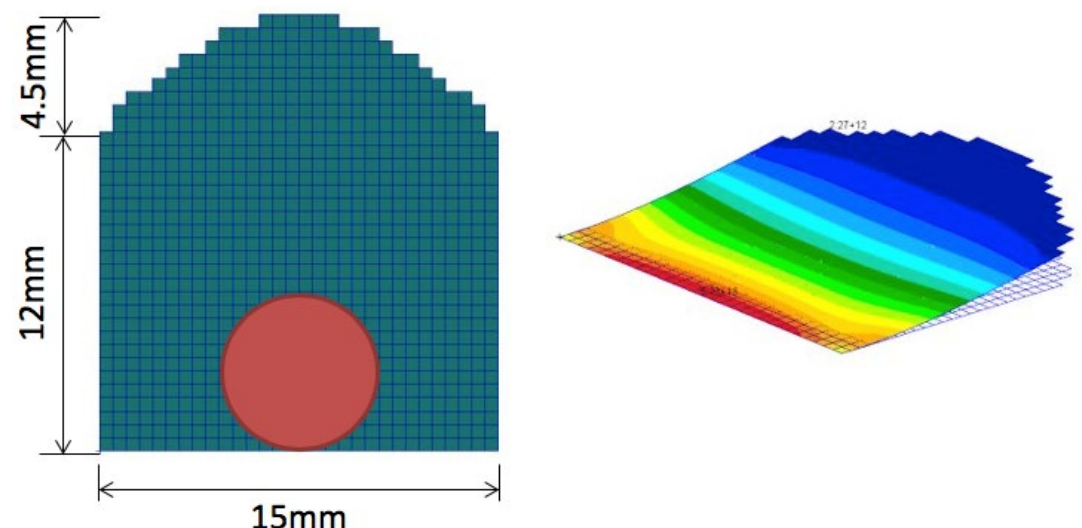

Figure 2. Finite element model and first mode shape of optimised cantilever for $1900 \mathrm{~Hz}$

To determine the performance of the energy harvesting device, the open circuit voltage can be used as a measure of the potential output power. The total electric charge $(Q)$ is mathematically defined as follows:

$$
Q=C V
$$

where $C$ is the equivalent capacitance and $V$ is the electric potential. Therefore, the open circuit voltage across the piezoelectric material can be found from:

$$
V_{o c}=\frac{1}{C} \iint[d] \boldsymbol{\sigma} \cdot d \boldsymbol{A}
$$

where $[d]$ is the piezoelectric strain tensor, $\boldsymbol{\sigma}$ is the mechanical stress vector and $\boldsymbol{A}$ is the area vector in the three planes (Fig. 1). In this work the finite element method (FEM) is used to solve the physical equations. 


\section{Experimental}

This section describes the fabrication process for the prototype harvester, including the waterjet cutting of the spring steel cantilever and the bonding of the PZT transducers. Also detailed are the experimental characterisations of the prototype harvester that have been undertaken, including impedance analysis of the PZT transducer, and voltage and power measurements as a function of drive acceleration and frequency, and load resistance.

\section{Energy Harvester Assembly}

As mentioned in section 2, the cantilevered section of the topology optimised energy harvester (Fig. 1) was fabricated using $0.51 \mathrm{~mm}$ thick carbon spring steel (AISI 1095, elastic modulus 190$210 \mathrm{GPa}$, Poisson's ratio $0.27-0.3$, density $7860 \mathrm{~kg} / \mathrm{m}^{3}$ ). The beams were cut using a CNC driven waterjet cutter (Techni). The waterjet pressure was $50 \mathrm{kPSI}$, and the diameter of the water beam was $0.5-0.8 \mathrm{~mm}$ with cutting grit (GMA garnet, composed of $>97 \%$ almandite garnet) located on the perimeter of the waterjet. The cantilevered section was secured between two spring steel root-plates that were each $0.89 \mathrm{~mm}$ thick. The root-plates were bonded to the cantilevered section using $0.2 \mathrm{~mm}$ thick polypropylene scrimcloth and a structural adhesive (Click Bond CB359).

Figure 1 shows the PZT disk transducer type chosen for the topology optimised harvester. The PZT disk has a $6.35 \mathrm{~mm}$ diameter and is $0.5 \mathrm{~mm}$ thick, and is a soft-relaxor (Pz27, Navy II PZT5A analogue, Ferroperm, Meggit A/S) with a piezoelectric charge constant, $\mathrm{d}_{31} \sim 150 \mathrm{pC} / \mathrm{N}^{\prime}$ and with an electromechanical coupling factor, $\mathrm{k}_{31} \sim 0.3$. The PZT disks were characterised using an impedance analyser (Solartron SI 1260) before and after bonding to the cantilevered section. A silver loaded epoxy (Chemtronics CW2400) was used to bond the PZT disks at high stress locations identified by FEA. During bonding a light pressure was applied in a circular motion to minimise the thickness of the epoxy layer beneath the disk with the goal of reducing damping on the piezoelectric disk [19] but increasing the presence of a spew fillet [20]. Thin copper wires were then bonded to the centre of each disk with the silver epoxy, and wire was additionally bonded on the exposed cantilever layer on the upper right side of the root to form a ground path.

\section{Harvester Characterisation}

The prototype harvester was mounted onto a host mass and a sinusoidal host acceleration was applied in the vertical ' 3 ' direction (Fig. 1) by an electro-dynamic shaker. Impedance frequency sweeps were conducted on the mounted harvester, yielding an estimate of the device's resonant frequency. Frequency sweeps were conducted during which the harvester's peak open circuit output voltage was recorded. The sweeps were repeated under a range of applied resistive loads with the associated voltage and power outputs determined. For vibrational testing, topologies were individually secured to the host mass, mounted on an electro-dynamic shaker (TIRA Vib Modal-Vibration Test System) using neodymium rare-earth magnets (N42, $50 \mathrm{~mm}$ x $5 \mathrm{~mm}$ x $3.4 \mathrm{~mm}$, partially visible in Fig. 3). The shaker arrangement is limited to drive levels equivalent to a maximum host acceleration of $2 g$ (where $g=9.81 \mathrm{~m} / \mathrm{s}^{2}$ ). 


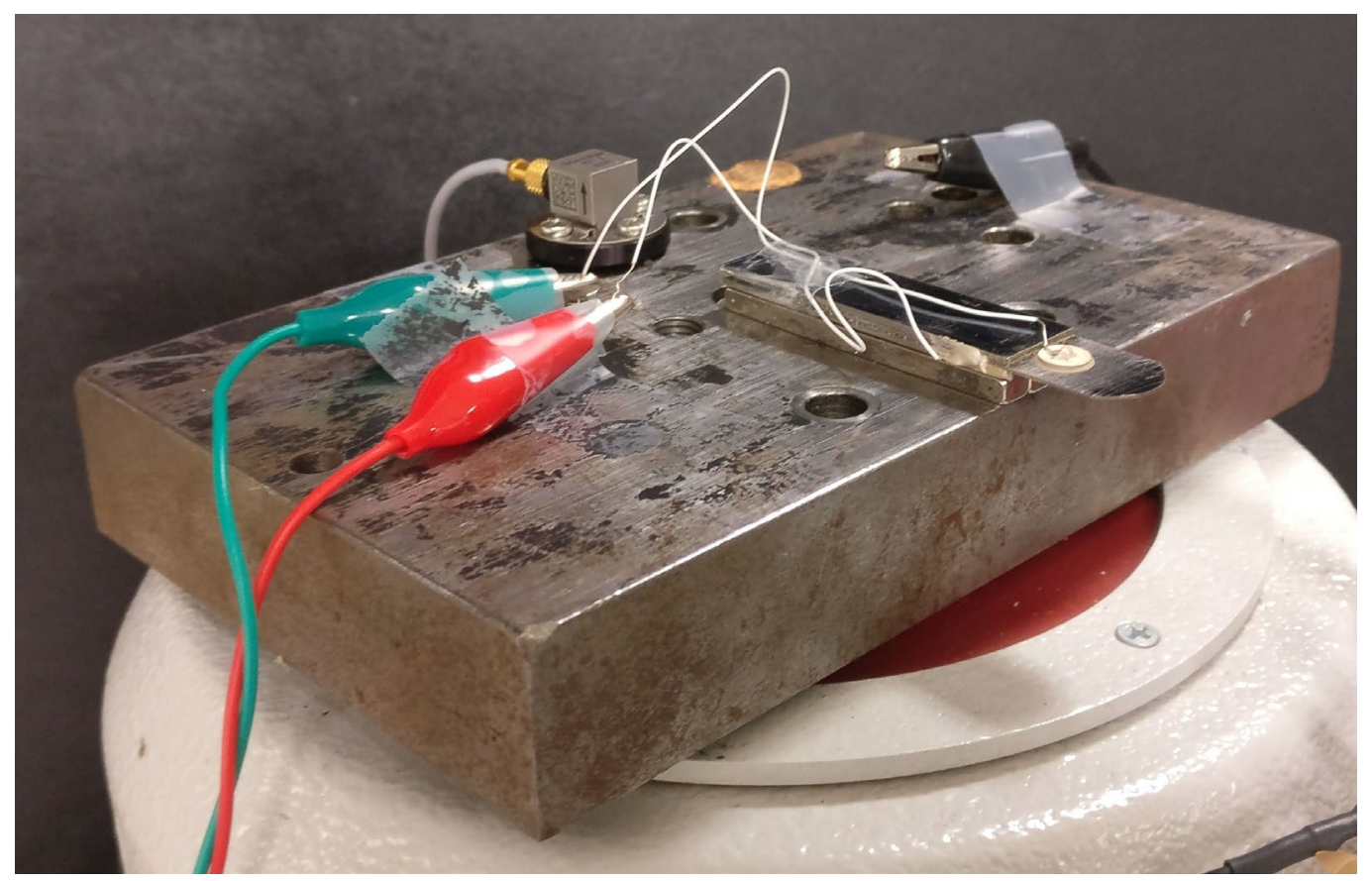

Figure 3. Photograph of the experimental arrangement showing the harvester mounted to the shaker/host mass with 'rails' magnet arrangement, and nearby accelerometer (cube geometry).

A loop controller (Brüel and Kjær 7541) was used to generate $0.2 g$ host vibrations over the frequency range of $1000 \mathrm{~Hz}$ to $3000 \mathrm{~Hz}$ with a sweep rate of $20 \mathrm{~Hz} / \mathrm{s}$. For higher drive levels, open loop experiments were performed using a signal generator, fed into an amplifier, to sweep the $1000-3000 \mathrm{~Hz}$ range at a sweep rate of $200 \mathrm{~Hz} / \mathrm{s}$. These open loop tests were performed at host accelerations of $1 g$ and $2 g$ with the drive levels set at the harvester's resonant frequency; however, the host acceleration could not be kept constant across all frequencies due to equipment limitations. The harvester's optimum load was determined using $0.2 g$ frequency sweeps and varying the load resistance in the range $10 \mathrm{k} \Omega$ to $150 \mathrm{k} \Omega$ (resistor decade box Tenma 72-7270). The same optimum load resistor was then used for sweeps at $1 g$ and $2 g$ to find the maximum output power.

\section{Results and Discussion}

This section will examine the frequency response of the harvester's impedance, compare model predictions and measured response of the harvesters open circuit output voltage as a function of frequency and host acceleration, and finally present measured resistor sweeps and measurements of output power versus frequency.

Figure 4 illustrates the measured impedance of a Pz27 disk bonded to the harvester cantilever arrangement shown in Fig. 3. The clamped capacitance of the bonded disk was $\sim 910 \mathrm{pF}$, with clamped relative permittivity 1620 . The $100 \mathrm{mV}$ drive voltage of the impedance analyser excited an electromechanical resonance near $1873 \mathrm{~Hz}$ with an amplitude of $80 \mathrm{k} \Omega$. This resistor value was used to set the approximate midpoint of the resistor range examined to find the optimum load (I.e. range $10 \mathrm{k} \Omega$ to $150 \mathrm{k} \Omega$ ). 


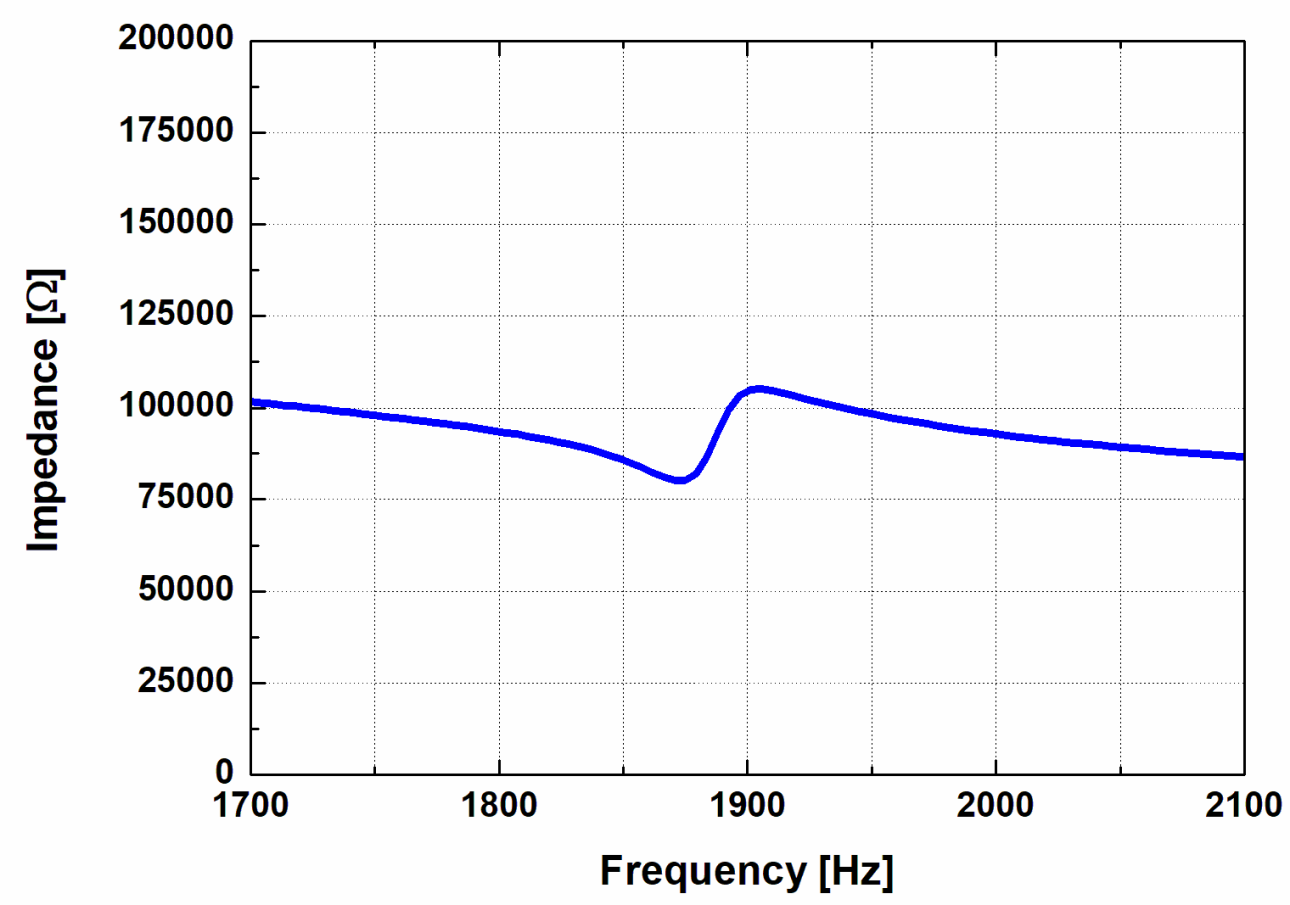

Figure 4. Measured impedance curve from a PZT disk mounted on a prototype harvester.

Figures $5 \mathrm{a}$ and $5 \mathrm{~b}$ show the predicted and measured open circuit harvester output voltage as a function of host frequency and acceleration, respectively. Both the predicted and measured results show the output voltage increasing linearly with host acceleration. Under all drive accelerations the resonant peaks occur just below the $1900 \mathrm{~Hz}$ design goal, with the peak voltage of $9 \mathrm{~V}$ predicted and observed for a $2 g$ host acceleration. There is a strong correlation between the predicted and measured harvester responses, indicating that the model contains sufficient physics to describe the device's open circuit behaviour, and that second order effects due to bondline thickness and spew fillet size are not significantly affecting the device behaviour. 

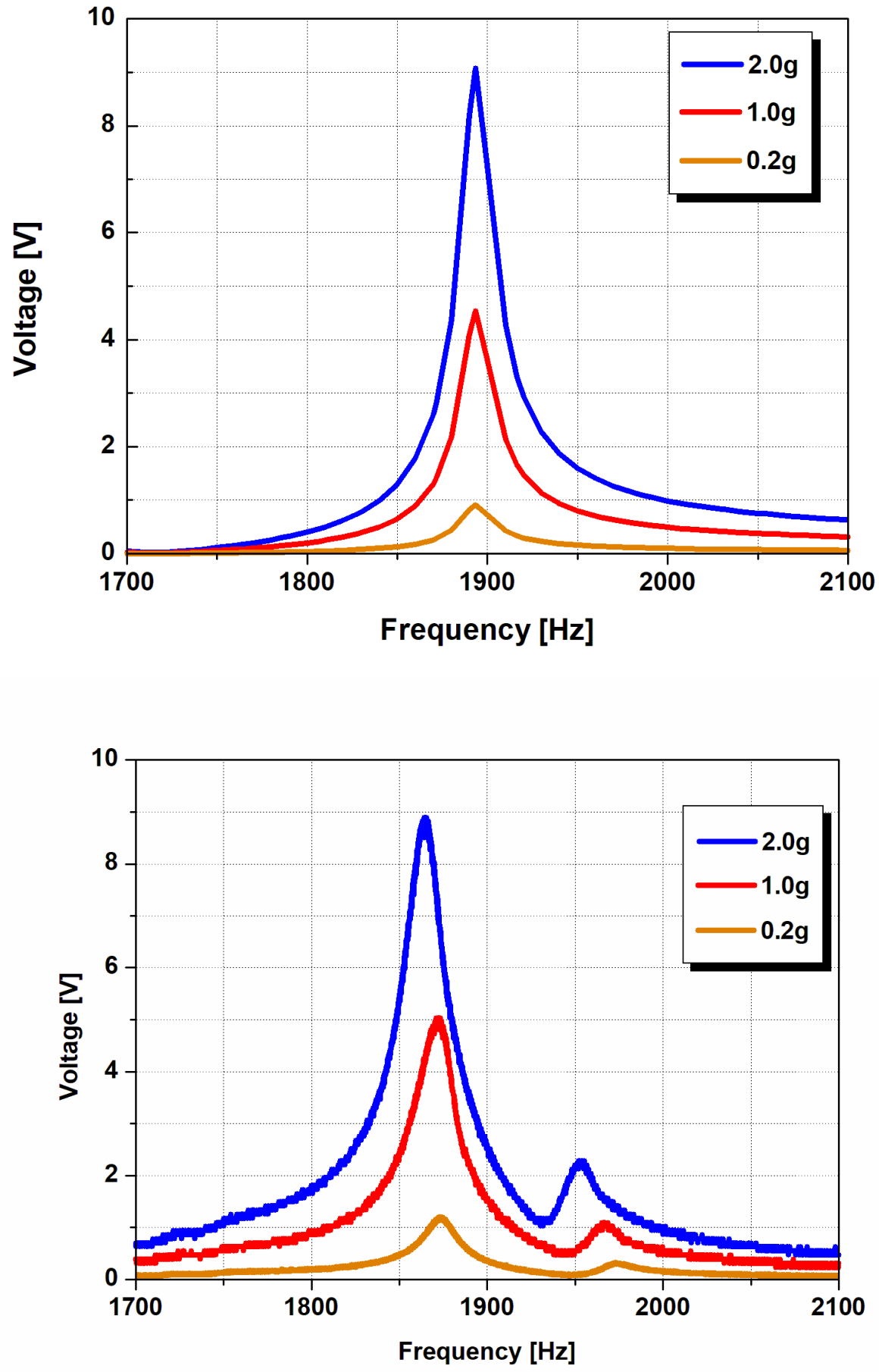

Figure 5. (a) Modelled open circuit voltage versus frequency, and (b) measured open circuit voltage versus frequency. 

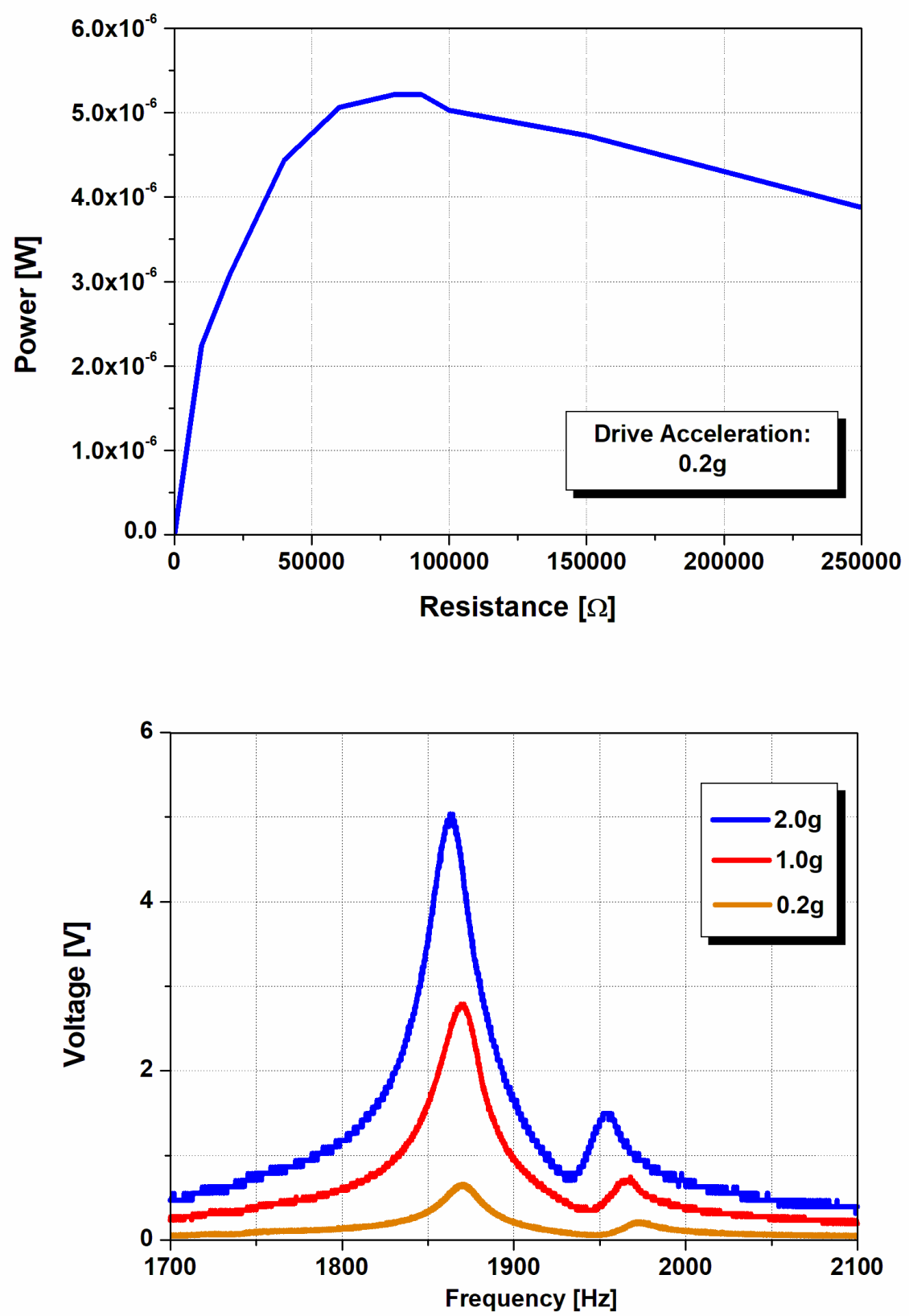

Figure 6. (a) Measured peak voltage and power versus resistive load. (b) Measured output voltage at optimum resistive load $85 \mathrm{k} \Omega$ and host excitations of $0.2 \mathrm{~g}$, $1 \mathrm{~g}$ and $2 \mathrm{~g}$.

Figure 6a shows the measured output power from the harvester as a function of resistive load, collected using $0.2 \mathrm{~g}$ closed-loop frequency sweeps. The maximum output power is $\sim 5.1 \mu \mathrm{W}$ under a host excitation of $0.2 \mathrm{~g}$ at $1870 \mathrm{~Hz}$, and occurs with a load of $85 \mathrm{k} \Omega$, which is close to the $80 \mathrm{k} \Omega$ impedance measured in Fig. 4. This suggests that an impedance sweep can provide a good estimate of optimum load, at least for devices based on traditional piezoceramics. There is a smaller resonance near $1950-1980 \mathrm{~Hz}$ that appears to be an artefact of the experimental 
arrangement and likely due to minor irregularities at the cantilever root-line of the three-layer structure of the device. Figure $6 \mathrm{~b}$ shows the measured output voltage from the harvester under an $85 \mathrm{k} \Omega$ resistive load, and as a function of frequency and drive acceleration. As might be expected the output voltages under electrical load are just over half of that measured under open circuit [21], with the resonant frequencies shifted 2-3 Hz lower. The peak output power under a $2 g$ host excitation was $\sim 300 \mu \mathrm{W}$, equivalent to a peak power density of $18.5 \mathrm{~mW} / \mathrm{cm}^{3}$, at the lower end of the $10-100 \mathrm{~mW} / \mathrm{cm}^{3}$ range discussed in the introduction. As mentioned earlier, the principal mesh frequency Bell 206B-1 Kiowa helicopter main rotor transmission occurs at $1900 \mathrm{~Hz}$ and is $12 \mathrm{~g}$. Under a $12 \mathrm{~g}$ excitation the harvester's output voltage might be expected to increase by a factor of 6 compared with the $2 g$ results presented here, up to around $30 \mathrm{~V}$. The electric field produced across the disk by this voltage is well below the coercive field of PZT-5A analogues, $\sim 1.2 \mathrm{kV} / \mathrm{mm}$ [22], hence is insufficient to induce depolarisation of the transducer element. Assuming a factor of 6 increase in output voltage, then the power might be expected to increase by a factor of 36 to around $11 \mathrm{~mW}$, which would be useful for powering many sensor types.

\section{Conclusion}

A topology optimised vibration energy harvester has been reported. The harvester is based on an optimised $0.51 \mathrm{~mm}$ thick spring-steel cantilever with a piezoceramic Pz27 disk transducer $6.35 \mathrm{~mm}$ diameter. The harvester is optimised to harvest from the principal gear-meshing frequency of a Bell 206B-1 Kiowa helicopter main rotor transmission which occurs at $1900 \mathrm{~Hz}$ and has an amplitude of $12 \mathrm{~g}$. Impedance frequency analysis of a prototype harvester indicated that the fundamental vibration mode of the device was located at $1870 \mathrm{~Hz}$ and at $80 \mathrm{k} \Omega$. Subsequent testing of the device using an electro-dynamic vibration shaker, under a host excitation of $0.2 \mathrm{~g}$ showed a resonance at $1870 \mathrm{~Hz}$ with an optimum load at $85 \mathrm{k} \Omega$, similar to that measured using impedance analysis. In the laboratory under a host excitation of $2 \mathrm{~g}$ and $1870 \mathrm{~Hz}$ the harvester produced $\sim 300 \mu \mathrm{W}$ with a power density of $18.5 \mathrm{~mW} / \mathrm{cm}^{3}$, suggesting that the device may generate up to $11 \mathrm{~mW}$ if mounted on a Bell 206B-1 Kiowa helicopter main rotor transmission.

\section{References}

[1] N. Rajic, S. Galea, Thermoelastic Stress Analysis and Structural Health Monitoring: An Emerging Nexus, Structural Health Monitoring. 14 (2015) 57-72.

https://doi.org/10.1177/1475921714548936

[2] A. Baker, N. Rajic, C. Davis, Towards a practical structural health monitoring technology for patched cracks in aircraft structure, Composites Part A: Applied Science and Manufacturing. 40 (2009) 1340-1352. https://doi.org/10.1016/j.compositesa.2008.09.015

[3] A. Erturk, D. J. Inman, Piezoelectric energy harvesting, Wiley, United Kingdom, 2011. https://doi.org/10.1002/9781119991151

[4] T. Ikeda, Fundamentals of Piezoelectricity, Oxford University Press, New York, 1996.

[5] Z. Yang, S Zhou, J Zu, D. Inman, High-Performance Piezoelectric Energy Harvesters and Their Applications, Joule. 2 (2018) 642-697. https://doi.org/10.1016/j.joule.2018.03.011

[6] T. F. Doughney, S. D. Moss, D. Blunt, W. Wang, H. J. Kissick, Relaxor ferroelectric transduction for high frequency vibration energy harvesting, Smart Mater. Struct. 28 (2019) 065011. https://doi.org/10.1088/1361-665X/ab15a5

[7] D. J. Munk, G. A. Vio, G. P. Steven, Topology and shape optimization methods using evolutionary algorithms: a review, Struct. Multidisc. Optim. 52 (2015) 613-631.

https://doi.org/10.1007/s00158-015-1261-9 
[8] M. P. Bendsoe, N. Kikuchi, Generating optimal topologies in structural design using homogenization, Comput. Methods Appl. Mech. Eng. 71 (1988) 197-224. https://doi.org/10.1016/0045-7825(88)90086-2

[9] J. D. Deaton, R. V. Grandhi, A survey of structural and multidisciplinary continuum topology optimization: post 2000, Struct. Multidisc. Optim. 49 (2014) 1-38.

https://doi.org/10.1007/s00158-013-0956-z

[10] O. Sigmund, K. Maute, Topology optimization approaches, Struct. Multidisc. Optim. 48 (2013) 1031-1055. https://doi.org/10.1007/s00158-013-0978-6

[11] G. I. N. Rozvany, A critical review of established methods of structural topology optimization, Struct. Multidisc. Optim. 37 (2009) 217-237. https://doi.org/10.1007/s00158-0070217-0

[12] N. Olhoff, Optimization of vibrating beams with respect to higher order natural frequencies, Journal of Structural Mechanics 4 (1976) 87-122. https://doi.org/10.1080/03601217608907283

[13] D. J. Munk, G. A. Vio, G. P. Steven, Novel moving isosurface threshold technique for optimisation of structures under dynamic loading, AIAA Journal, 55 (2017) 638-651.

https://doi.org/10.2514/1.J054692

[14] F. Goldschmidtboeing, P. Woias, Characterization of different beam shapes for piezoelectric energy harvesting, Journal of Micromechanics and Microengineering, 18 (2008).

https://doi.org/10.1088/0960-1317/18/10/104013

[15] D. J. Munk, A bidirectional evolutionary structural optimization algorithm for mass minimization with multiple structural constraints, International Journal for Numerical Methods in Engineering, 118 (2019) 93 - 120. https://doi.org/10.1002/nme.6005

[16] X. Huang, Y. M. Xie, Bi-directional evolutionary topology optimization of continuum structures with one or multiple materials, Computational Mechanics, 43 (2009) 393 - 401. https://doi.org/10.1007/s00466-008-0312-0

[17] O. Sigmund, J. Petersson, Numerical instabilities in topology optimization: a survey on procedures dealing with checkerboards, mesh-dependencies and local minima, Structural Optimization, 16 (1998) 291 - 299. https://doi.org/10.1007/BF01214002

[18] M. P. Bendsoe, O. Sigmund, Topology Optimization: Theory, Methods and Applications, 1st Edition (2003) Springer Berlin. https://doi.org/10.1007/978-3-662-05086-6_2

[19] T. J. Johnson, D. Charnegie, W. W. Clark, Energy harvesting from mechanical vibrations using piezoelectric cantilever beams, Proc. of SPIE. 6169 (2006) 61690D.

https://doi.org/10.1117/12.659466

[20] S. D. Moss, S. C. Galea, I. G. Powlesland, M. Konak, A. A. Baker, In-situ health monitoring of a bonded composite patch using the strain ratio technique, Proc. SPIE, 4235 (2001) 363-374. https://doi.org/10.1117/12.420878

[21] S. Roundy, P. K. Wright, J. M. Rabaey, Energy Scavenging for Wireless Sensor Networks with Special Focus on Vibrations, Springer, Boston, 2004. https://doi.org/10.1007/978-1-46150485-6

[22] M. W. Hooker, Properties of PZT-Based Piezoelectric Ceramics Between -150 and 250C, NASA/CR-1998-208708, Langley Research Center, 1998. 